\title{
A TAUBERIAN GROUP ALGEBRA
}

\author{
PETER R. MUELLER-ROEMER
}

Abstract. Let $G$ be the group of real matrices

$$
(x, y)=\left(\begin{array}{ll}
e^{x} & 0 \\
y & 1
\end{array}\right) \quad(x, y \in R) .
$$

Every proper closed two-sided ideal of $L^{1}(G)$ is contained in a maximal modular two-sided ideal. The strong radical of $L^{1}(G)$ is the set of all $f \in L^{1}(G)$ with $\int f(x, y) d y=0$ for almost all $x \in R$. The strong structure spaces of $L^{1}(G)$ and $L^{1}(R)$ are homeomorphic.

Call a Banach algebra $A$ tauberian if every proper closed two-sided ideal of $A$ is contained in a maximal modular two-sided ideal. For completely regular Banach algebras this definition coincides with Rickart's (cf. [2, 2.7.25]). The $L^{1}$-group algebras of compact and of locally compact abelian groups are known to be tauberian. Probably, it is already known that the direct product of a compact and an abelian group has a tauberian group algebra. A quite different example follows.

Let $G$ be the group of real matrices $(x, y)=\left(\begin{array}{ll}e^{x} & 0 \\ y & 1\end{array}\right)(x, y \in \boldsymbol{R})$ with its natural topology, and let $H \cong \boldsymbol{R}$ be the normal subgroup of elements $(0, y)$. The law of composition in $G$ is

$$
(x, y)(u, v)=\left(x+u, e^{u} y+v\right)
$$

and thus $d(u, v)=d u d v$ is the (left) Haar measure on $G$. Moreover,

and

$$
(u, v)^{-1}=\left(-u,-e^{-u} v\right)
$$

$$
(u, v)^{-1}(x, y)(u, v)=\left(x,\left(1-e^{x}\right) v+e^{u} y\right) .
$$

The convolution product of $f$ and $g$ in $L^{1}(G)$ is given by

$$
f * g(x, y)=\int f\left(x+u, e^{u} y+v\right) g\left(-u,-e^{-u} v\right) d u d v
$$

Presented to the Society, September 3, 1971 under the title $A$ noncommutative, noncompact group with tauberian group algebra; received by the editors July 22, 1971 and, in revised form, November $15,1971$.

AMS (MOS) subject classifications (1970). Primary 43A20, 46H10; Secondary 43A65, 43A80, 16A21, 16A66.

Key words and phrases. Tauberian, completely regular algebra, strong radical, group algebra, amenable group.

c, American Mathematical Society 1973 
and the canonical projection

$$
T_{H}: L^{1}(G) \rightarrow L^{1}(G / H) \cong L^{1}(R)
$$

by $\left(T_{H} f\right)(x)=\int f(x, y) d y$. Its kernel will be denoted by $K=T_{H}^{-1}(0)$. Let $j_{0}$ be the kernel of the trivial character of $L^{1}(R)$, i.e. $j_{0}=\left\{q \in L^{1}(R) \mid \hat{q}(0)=\right.$ $\left.\int q(y) d y=0\right\}$. Now $K$ can easily be identified with $L^{1}\left(\boldsymbol{R}, j_{0}\right)$, which in turn contains $L^{1}(\boldsymbol{R}) \times j_{0}$ as a total subset. In other words, the elements of $K$ can be approximated by finite sums of elements $p \otimes q-p \otimes q(x, y)=p(x) q(y)-$ with $p$ and $q$ from $L^{\mathbf{1}}(\boldsymbol{R})$ and $\hat{q}(0)=\int q(y) d y=0$.

The element $(-\log z, 0)$ for $z>0$ defines the inner automorphism $i_{z}$ of $G$,

$$
i_{z}(x, y)=\left(x, z^{-1} y\right)
$$

and $i_{z}$ induces an isometric automorphism $M_{z}$ of $L^{1}(G)$ given by

$$
\left(M_{z} f\right)(x, y)=z^{-1} \cdot f\left(x, z^{-1} y\right) \text { for } f \in L^{1}(G) .
$$

Similarly,

$$
\left(M_{z} f\right)(y)=z^{-1} f\left(z^{-1} y\right)
$$

defines an isometric automorphism of $L^{1}(\boldsymbol{R})$. By [1, Chapter 1, $\S 2.2$, p. 6],

$$
\lim _{z \rightarrow 0}\left(M_{z} f\right) * g=\hat{f}(0) g \text { for } f, g \in L^{1}(\boldsymbol{R}) .
$$

REMARK 1. This property is essential to the main proof and seems to have no analogue in general locally compact groups.

$$
\lim _{z \rightarrow 0}\left(M_{z} f\right) * g=0 \text { for } f \in K, g \in L^{1}(G) .
$$

Proof. It suffices to prove (2) for $f=p \otimes q$ with $p, q \in L^{1}(\boldsymbol{R}), \hat{q}(0)=0$ and $g=h \otimes k$ with $h, k$ continuous functions with compact support, since these functions are total in $K$ and $L^{1}(G)$ respectively. Now $M_{z} f=p \otimes M_{z} q$ and

Consequently

$$
\begin{aligned}
\left(M_{z} f * g\right)(x, y) & =\int p(x+u)\left(M_{z} q\right)\left(e^{u} y+v\right) h(-u) k\left(-e^{-u} v\right) d u d v \\
& =\int p(x+u) h(-u) \int e^{u}\left(M_{z} q\right)\left(e^{u}(y+v)\right) k(-v) d v d u \\
& =\int p(x+u) h(-u)\left(\left(M_{c^{-u} z} q\right) * k\right)(y) d u .
\end{aligned}
$$

$$
\left|M_{z} f * g\right|_{1} \leqq \int|p(x+u)||h(-u)|\left|\left(M_{c^{-u_{z}}} q\right) * k\right|_{1} d x d u
$$

The support of $h$ is contained in an interval $\left[-a,+\dot{a}^{\prime}\right]$ and, by (1), $\left|M_{t} q * k\right|_{1} \leqq \varepsilon$ for $t \leqq \delta=\delta(\varepsilon)$. Now $e^{-u} z \leqq \delta$ for $|u| \leqq a$ and $z \leqq e^{-a} \delta$; hence 
we obtain

$$
\begin{aligned}
\left|M_{z} f * g\right|_{1} & \leqq|p|_{1} \int_{-a}^{+a}|h(-u)|\left|\left(M_{e^{-u} z} q\right) * k\right|_{1} d u \\
& \leqq \varepsilon|p|_{1} \cdot|h|_{1} \quad \text { for } z \leqq e^{-a} \delta
\end{aligned}
$$

REMARK 2. Let $f=p \otimes q$ with $p, q$ in $L^{1}(R), q \geqq 0$ and $\hat{q}(0)=1$, and let $g=h \otimes k$ be as above. A similar computation leads to

$$
\left|M_{z} f * g-g\right|_{1} \leqq|p|_{1}|h|_{1} \sup _{|u| \leqq a}\left|\left(M_{e^{-u}} q\right) * k-k\right|_{1}+|p * h-h|_{1} \cdot|k|_{1} \text {. }
$$

Again, the supremum can be made arbitrarily small, and if $p$ is chosen conveniently from an approximate identity $\left\{p_{i}\right\}_{i}$ the second term becomes small. Also $\left|M_{z} f\right|_{1}=\left|p_{i}\right|_{1} \cdot \hat{q}(0)=1$. Thus a density argument for the $g$ 's shows that $\left\{p_{i} \otimes M_{z} q\right\}_{(i, z)}$ is an approximate identity for $L^{1}(G)$ if $(i, z) \geqq$ $\left(j, z^{\prime}\right)$ is defined to mean $i \geqq j$ and $z \leqq z^{\prime}$.

THEOREM. If $J$ is a proper closed two-sided ideal in $L^{1}(G)$, then so is $J+K$.

Proof. By $\S 4.6(i i)$, Chapter 8 in [1] $J+K$ is a closed, two-sided ideal. We will show that if $J+K=L^{1}(G)$ then $J=L^{1}(G)$. Let $f \in L^{1}(G)$ and let $\left\{p_{i} \otimes M_{z} q\right\}_{(i, z)}$ be the approximate identity described in Remark 2. Set $q_{i}=p_{i} \otimes q$ and $q_{i, z}=p_{i} \otimes M_{z} q=M_{z} q_{i}$. Since $J+K=L^{1}(G), q_{i}=q_{i}^{\prime}+q_{i}^{\prime \prime}$ with $q_{i}^{\prime} \in J, q_{i}^{\prime \prime} \in K$. Since $J$ and $K$ are invariant under left translations $L_{g}$, $\left(L_{g} f\right)\left(g^{\prime}\right)=f\left(g^{-1} g^{\prime}\right)$ and right translations $R_{g},\left(R_{g} f\right)\left(g^{\prime}\right)=\Delta(g) f\left(g^{\prime} g\right)$, they are invariant under $M_{z}=L_{g} R_{g}$, with $g=(-\log z, 0)$, and it follows that $q_{i, z}=q_{i, z}^{\prime}+q_{i, z}^{\prime \prime}$ with $q_{i, z}^{\prime}=M_{z} q_{i}^{\prime} \in J, q_{i, z}^{\prime \prime}=M_{z} q_{i}^{\prime \prime} \in K$. For $\varepsilon>0$ there exists $\left(i_{0}, z_{0}\right)$ such that $\left|f-q_{i, z} * f\right|_{1}<\varepsilon$ provided $(i, z) \geqq\left(i_{0}, z_{0}\right)$, i.e. provided $i \geqq i_{0}$ and $z \leqq z_{0}$. Consequently,

$$
\left|f-q_{i, z}^{\prime} * f\right|_{1} \leqq \varepsilon+\left|q_{i, z}^{\prime \prime} * f\right|_{1}
$$

Since $q_{i}^{\prime \prime} \in K,(2)$ implies

i.e.

$$
\lim _{z \rightarrow 0}\left|q_{i . z}^{\prime \prime} * f\right|_{1}=\lim _{z \rightarrow 0}\left|M_{z} q_{i}^{\prime \prime} * f\right|_{1}=0
$$

Hence

$$
\left|q_{i, z}^{\prime \prime} * f\right|_{1} \leqq \varepsilon \quad \text { for } z \leqq \delta(i, \varepsilon) \leqq z_{0}
$$

$$
\left|f-q_{i, z}^{\prime} * f\right|_{1} \leqq 2 \varepsilon \quad \text { for } i \geqq i_{0}, z \leqq \delta(i, \varepsilon) .
$$

Since $\varepsilon$ was arbitrary, this implies $f \in J$.

COROllary 1. The maximal modular two-sided ideals of $L^{1}(G)$ contain $K$.

Proof. If $J$ is a maximal modular two-sided ideal in $L^{1}(G), J+K$ is a proper modular two-sided ideal containing $J$. By maximality $J=J+K \supset K$. 
Remark 3. Not all closed two-sided ideals of $L^{1}(G)$ contain $K$ : Let $j_{+}\left[j_{-}\right]$be the ideal in $L^{1}(R)$ which consists of the functions $g$ whose Fourier transforms $\hat{g}$ vanish for all positive [negative] $\lambda$, i.e.

$$
\hat{g}(\hat{\lambda})=\int e^{i \lambda y} g(y) d y=0 \quad(\lambda>0[\lambda<0]) .
$$

By continuity $\hat{g}(0)=0$; hence $j_{ \pm} \subset j_{0}$. Let $J_{+}$be the set of $f \in L^{1}(G)$ with $(y \rightarrow f(x, y)) \in j_{+}$for almost all $x$. Let $J_{-}$be similarly defined. By the uniqueness of the Fourier transform $j_{+} \cap j_{-}=\{0\}$, hence also $J_{+} \cap J_{-}=\{0\}$. $J_{+} \oplus J_{-} \subset K$ and neither $J_{+}$nor $J_{-}$contains $K$. That $J_{+}$and $J_{-}$are closed twosided ideals can be seen as follows: The operator $A_{\lambda}$ defined by $\left(A_{\lambda} f\right)(x, y)=$ $e^{i \lambda y} f(x, y)$ is an isometry of $L^{1}(G)$ and thus maps closed subspaces into closed subspaces. In particular $K_{\lambda}=A_{\lambda}^{-1} K$ and $J_{+}=\bigcap_{\lambda>0} K_{\lambda}$ are closed. It is easy to check that $K_{\lambda}$.is invariant under left-translations and that the right-translation $R_{(a, b)}$ maps $K_{\lambda}$ onto $K_{\lambda e^{-a}}$. Hence, $J_{+}$is a two-sided ideal.

COROLlaRY 2. $T_{H}$ induces a homeomorphism of the strong structure spaces of $L^{1}(G)$ and $L^{1}(R)$ and $K$, the kernel of $T_{H}$, is the strong radical of $L^{1}(G)$.

Proof. By $\S 4.4$, Chapter 3 of [1] the isomorphism $L^{1}(G / H) \cong L^{1}(G) / K$ is algebraic and isometric. By Theorem 2.6.6 of [2] and Corollary 1 the strong structure spaces of $L^{1}(R) \cong L^{1}(G / H)$ and $L^{1}(G)$ are homeomorphic. Since $L^{1}(\boldsymbol{R})$ is strongly semisimple its strong radical is $\{0\}$, and the inverse image $K$ of $\{0\}$ under $T_{H}$ is the strong radical of $L^{1}(G)$.

COROLlaRY 3. $L^{1}(G)$ is a completely regular tauberian Banach algebra.

Proof. By the theorem, $T_{H}(J) \cong(J+K) / K$ is a proper closed two-sided ideal iff $J$ is one. Since every such ideal $T_{H}(J)$ is contained in a maximal modular two-sided ideal $M$ of $L^{1}(R), J$ is contained in $T_{H}^{-1}(M)$ which is itself modular. Thus $L^{1}(G)$ is tauberian (cf. 2.7 .25 of [2]). Since $L^{1}(R)$ is completely regular 2.7 .4 of [2] implies that $L^{1}(G)$ is completely regular.

ACKNOWLEDGMENT. The author is very grateful to Horst Leptin who suggested this problem, read the first draft of this paper very carefully and who permitted the author to reproduce his more concise version of the proof of the theorem.

\section{BIBLIOGRAPHY}

1. H. Reiter, Classical harmonic analysis and locally compact groups, Oxford Univ. Press, Oxford, 1968.

2. C. E. Rickart, General theory of Banach algebras, University Series in Higher Math., Van Nostrand, Princeton, N.J., 1960. MR 22 \#5903.

Department of Mathematics, East Carolina University, Greenville, North CAROLINA 27834 